Return to the Manage Active Submissions page at http://spie.org/app/submissions/tasks.aspx and approve or disapprove this submission. Your manuscript will not be published without this approval. Please contact author_help@spie.org with any questions or concerns.

\title{
A comparison of two global optimization algorithms with sequential niche technique for structural model updating
}

\author{
${ }^{1}$ Faisal Shabbir, ${ }^{2}$ Piotr Omenzetter ${ }^{*}$ \\ ${ }^{1}$ Department of Civil and Environmental Engineering, The University of Engineering and \\ Technology, Taxila, Pakistan. \\ ${ }^{2}$ The LRF Centre for Safety and Reliability Engineering, The University of Aberdeen, Aberdeen, \\ UK.
}

\begin{abstract}
Much effort is devoted nowadays to derive accurate finite element (FE) models to be used for structural health monitoring, damage detection and assessment. However, formation of a FE model representative of the original structure is a difficult task. Model updating is a branch of optimization which calibrates the FE model by comparing the modal properties of the actual structure with these of the FE predictions. As the number of experimental measurements is usually much smaller than the number of uncertain parameters, and, consequently, not all uncertain parameters are selected for model updating, different local minima may exist in the solution space. Experimental noise further exacerbates the problem. The attainment of a global solution in a multi-dimensional search space is a challenging problem. Global optimization algorithms (GOAs) have received interest in the previous decade to solve this problem, but no GOA can ensure the detection of the global minimum either. To counter this problem, a combination of GOA with sequential niche technique (SNT) has been proposed in this research which systematically searches the whole solution space. A dynamically tested full scale pedestrian bridge is taken as a case study. Two different GOAs, namely particle swarm optimization (PSO) and genetic algorithm (GA), are investigated in combination with SNT. The results of these GOA are compared in terms of their efficiency in detecting global minima. The systematic search enables to find different solutions in the search space, thus increasing the confidence of finding the global minimum.
\end{abstract}

Keywords: finite element method, modal data, model updating, particle swarm optimization, genetic algorithm, sequential niche technique.

\section{INTRODUCTION}

A highly developed infrastructure is vital for economic growth in this modern age. The actual behavior of structures has often been shown to be much different from that considered in a specification-based design approach ${ }^{1}$. In addition, the degradation and deterioration of civil infrastructure is unfortunately inevitable due to many factors, including harsh operational conditions, physical aging and natural as well as man-made hazards. ${ }^{2}$. Important components of the infrastructure should maintain their service life and be able to perform well in typical operating conditions as well as in extreme events. It is therefore important to determine the structural characteristics of infrastructural components so as to correctly predict their response ${ }^{3}$. In recent years, the investigation and characterization of constructed civil engineering facilities has gained much attention ${ }^{4,5}$. A relatively high cost associated with the maintenance of the existing infrastructure is the primary motivating factor. Accurate assessment of the condition of existing infrastructure is therefore imperative to make optimal decisions for asset management ${ }^{4-6}$.

For the design and analysis of civil engineering systems, the finite element (FE) method is widely used. FE models of civil structures are usually based on idealized drawings/designs and estimates of material properties, structural geometry and boundary and connectivity conditions, which may not truly reflect the exact behavior of the as-built system. Field tests can be performed on the full-scale structures to validate the FE models. The dynamic behavior of structures under external excitation is of major interest to structural engineers, as the experimental responses (such as modal frequencies,

* piotr.omenzetter@abdn.ac.uk; phone 44-1224-272529; fax 44-1224-272497; www.abdn.ac.uk 
Return to the Manage Active Submissions page at http://spie.org/app/submissions/tasks.aspx and approve or disapprove this submission. Your manuscript will not be published without this approval. Please contact author_help@spie.org with any questions or concerns.

damping and shapes) are related to physical properties of the system (mass, stiffness and energy dissipation). Significant differences in the dynamic behavior of FE models and as-built systems have been noted by various researchers ${ }^{7-9}$ and numerous studies focusing on damage detection and estimation have been carried out using vibration data ${ }^{10,11}$. Dynamic model updating is a process of refining a mathematical model of an actual structure using test measurements.

Contemporary model updating methods use the sensitivity method (SM) which gives a single updated solution ${ }^{8}$ but has a tendency to converge to local minima ${ }^{12}$. Although model updating using SM-based optimization has been well documented, the application of global optimization algorithms (GOAs) in this field have received limited attention.

Most recent studies which report GOA-based model updating are for damage detection of laboratory-scaled structures. Genetic algorithm (GA) was used ${ }^{13}$ for assessment and damage detection of a simulated beam structure and an experimental beam structure. Multiple damage scenarios were studied along with the effect of different noise levels on the simulated beam structure. Later, GA was applied to the laboratory beam structure to verify its effectiveness in the damage detection and its assessment. Raich and Liszkai ${ }^{14}$ presented an advanced GA and applied it to simulated beam and frame structures for improving the performance of damage detection. In another study ${ }^{15}$, both Simulated Annealing (SA) and GA were investigated to update a numerical model of a cantilever beam and an experimental wing plate structure. Saada et al. ${ }^{16}$ used particle swarm optimization (PSO) for model updating of a beam structure. A hybrid PSOSimplex method was proposed for model updating of a ten-bar truss and a free-free beam ${ }^{17}$. The new method proposed performed well for model updating of the numerically simulated structures. However, it can be noticed that most research efforts using GOAs have been made towards damage detection and assessment of simulated structures or simple laboratory scale structures. Although GOAs try to search the global minimum, the results are strongly dependent on different factors such as search capabilities of the algorithm, limited time and computational load and complexity of the search space .Therefore, the detection of global minimum from a multi-dimensional search space could not be assured using GOAs. To alleviate this problem, a combination of sequential niche technique (SNT) with GOAs is proposed in this study. Moreover, updating of full scale structures still remains a challenging and relatively unexplored topic. In this paper, two GOAs, namely PSO and GA combined with SNT have been explored for dynamic FE model updating of a full scale dynamically tested bridge.

In the sections to follow, theory related to PSO, GA, SNT and objective functions in model updating is detailed. Then the description of full scale bridge and its dynamic testing results are presented. PSO and GA combined with SNT were then applied for model updating of a numerical model of the full scale bridge to match the actual dynamic testing results with FE model counterparts. Finally, the updated results obtained are physically justified.

\section{THEORY}

\subsection{Particle swarm optimization}

$\mathrm{PSO}^{18}$ is a population-based stochastic optimization method that tries to improve the solution iteratively with respect to a given measure of quality. The concept of PSO was developed based on the swarming behavior of fish, bees and other animals. The basic idea is that if one of the members sees a desirable path for the most fertile feeding locations, the rest of the swarm should follow. Each particle in the swarm should be influenced by the rest of the swarm to increase the diversity but also be able to independently explore its own vicinity to a certain extent. Two factors mainly contribute to swarm intelligence: group knowledge and individual knowledge. This is achieved by the particles that have a position and velocity vector in multidimensional space, where each position coordinate represents a parameter to be optimized. The particles can have continuous or discrete values. PSO calculates the fitness of each particle from its fitness function. These particles flying in ' $n$ ' dimensional space have two reasoning capabilities: the memory of their own best position in consecutive generations called pbest $_{i}$, and knowledge of the swarm's best position normally called gbest. The velocity of each particle $\mathbf{v}_{i}$ at time $t$ towards its pbest ${ }_{i}$ and gbest locations is adjusted by the formula:

$$
\mathbf{v}_{i}(t+1)=\gamma \mathbf{v}_{i}(t)+c_{1} \text { rand }_{1}\left(\operatorname{pbest}_{i}(t)-\mathbf{x}_{i}(t)\right)+c_{2} \operatorname{rand}_{2}\left(\operatorname{gbest}(t)-\mathbf{x}_{i}(t)\right)
$$

where $\mathbf{x}_{i}$ is the particle position, $\gamma$ is the inertial weight, $c_{1}$ and $c_{2}$ are the cognition and social component, respectively, and rand $_{1}$ and rand $_{2}$ are random numbers uniformly distributed between 0 and 1 . 
Return to the Manage Active Submissions page at http://spie.org/app/submissions/tasks.aspx and approve or disapprove this submission. Your manuscript will not be published without this approval. Please contact author_help@spie.org with any questions or concerns.

\subsection{Genetic algorithm}

$\mathrm{GA}^{19}$ mimic the process of natural evolution according to Darwin's theory. GA works on a population of potential solutions based on the principle of survival of the fittest to produce a better and better solution. New approximations of the solution are created by selecting individuals according to their level of fitness and then breed together using the operators working on the concept of natural genetics. As the generations proceed, the whole process leads to the development of better suited individuals as compared to the individuals they are created from.

To form new solutions for the next generation, genetic operators are applied to the previously selected pool. The consistency of GA strongly depends on the relevant reproduction operators. If the reproduction operators just produce new solutions without any strong link to the ones in the previous generations, they are essentially performing a random search. The recombination operator normally exchanges genetic information between pairs of individuals. Most commonly used recombination operator is cross over where two chromosomes are cut and the halves thus obtained are spliced to form new chromosomes. This is a very important operator as it combines the characteristics from two parents into one chromosome. The cross over operator is not necessarily applied to all the individuals in a population and is applied with selected probability. The new solutions created typically share the characteristics of its parents. Another genetic operator called mutation is then applied to the new population. Mutation changes the individual genetic representation again according to a probabilistic rule. In binary string representation, mutation causes the single bit to change its value from 0 to 1 or vice versa. It is a background operator and generally ensures searching occurs over the entire solution domain. This has the effect of restraining the algorithm to converge to the local optima. After the application of recombination and mutation operators, the fitness value of each individual is again evaluated. The process continues to form new populations that are different from the initial generation. Since only the best particles are selected, this leads to a population of individuals that are better suited for minimizing the objective function. The average performance of the population increases as better individuals are preserved and less fit individuals perish.

\subsection{Sequential niche technique}

The principle of SNT is to carry over knowledge gained during subsequent iterations of an optimization algorithm ${ }^{20}$ so that different minima are discovered in turn. The basic idea behind SNT is that when a minimum is found in the search domain, the surrounding area, referred to as niche, is 'filled in' and no longer attracts the particles in subsequent iterations. This forces the optimization algorithm to converge to another, yet unvisited, niche. The process continues until the criteria such as the maximum number of iterations, maximum number of discovered minima and the upper threshold value of the objective function at a minimum have been met.

Initial iterations in search of the first minimum are made with the basic search algorithm without SNT by using the raw objective function. Once the first minimum has been found, the objective function values of the particles in the vicinity of the minimum are modified, and the search for the next minimum commences. The modifications to the objective function are introduced by multiplying it by a derating function using the following recursive formula:

$$
\Pi_{n+1}=\Pi_{n}(\mathbf{x}) \times G\left(\mathbf{x}, \mathbf{s}_{n}\right)
$$

where $\prod_{n+1}$ is the modified objective function to be used for searching for the $\mathrm{n}+1$-th minimum, $\prod_{n}$ is the previous objective function used for searching for the n-th minimum, $G\left(\mathbf{x}, \mathbf{s}_{n}\right)$ is the derating function, and $\mathbf{s}_{n}$ is the $\mathrm{n}$-th found minimum.

The following exponential derating function is used in this study ${ }^{20}$ :

$$
G\left(\mathbf{x}, \mathbf{s}_{n}\right)=\left\{\begin{array}{cc}
\log m \times \frac{r-d\left(\mathbf{x}, \mathbf{s}_{n}\right)}{r} & \text { if } d\left(\mathbf{x}, \mathbf{s}_{n}\right)<r \\
1 & \text { otherwise }
\end{array}\right.
$$

where $m$ is the parameter used to control concavity of the derating function, $r$ is the niche radius, and $d\left(\mathbf{x}, \mathbf{s}_{n}\right)$ defines the distance between the current point $\mathbf{x}$ and best individual $\mathbf{s}_{n}$.

\subsection{Objective function for model updating}

A combined objective function related to the frequencies and model assurance criterion ${ }^{21}$ (MAC) is used in this study. The relative error between the experimental and analytical frequencies is: 
Return to the Manage Active Submissions page at http://spie.org/app/submissions/tasks.aspx and approve or disapprove this submission. Your manuscript will not be published without this approval. Please contact author_help@spie.org with any questions or concerns.

$$
\Pi_{1}=\sum_{i=1}^{n}\left[\left(\omega_{a, i}-\omega_{e, i}\right) / \omega_{e, i}\right]^{2}
$$

where $\omega$ represents modal frequency, subscripts $a$ and $e$ refer to the analytical and experimental values, respectively, and $n$ is the total number of modes to be updated. The second objective function is related to the difference in mode shapes and can be defined in terms of MAC as:

$$
\Pi_{2}=\sum_{i=1}^{n}\left(1-\sqrt{M A C_{i}}\right)^{2} / M A C_{i}
$$

The combined objective function $\Pi$ is defined as follows:

$$
\Pi=\Pi_{1}+\Pi_{2}
$$

\section{THE BRIDGE, ITS MODEL AND DYNAMIC TESTING}

The full scale bridge under study is a $59,500 \mathrm{~mm}$ long cable stayed footbridge with two symmetrical spans separated by a central A-shaped pylon over a four-lane motorway. The bridge is shown in Figure 1 and basic dimensions are provided in Figure 2. The bridge deck is a trapezoidal steel girder with cast in-situ concrete slab of thickness $130 \mathrm{~mm}$. The deck is continuous over the entire span and is supported by a total of six pairs of stay cables spaced at approx. $8 \mathrm{~m}$ center to center. Different post-tension forces have been applied to each pair of cables ranging from $55 \mathrm{kN}$ to $95 \mathrm{kN}$ as shown in Figure 2. The cables were connected to the top of the pylon, which is composed of two steel I-sections joined with cross bracing that also supports the deck. The size of the pylon I-section is $400 \mathrm{WC} 328^{22}$ and the diameter of the cables used is $32 \mathrm{~mm}$. A closed rectangular pipe having a section of $250 \times 150 \times 9 \mathrm{~mm}$ also runs on both sides of the bridge deck and this encloses two $100 \mathrm{~mm}$ ducts for service pipes, with surrounding void spaces grouted with cement. A railing, which has a total height of $1400 \mathrm{~mm}$, is provided on both sides of the bridge. The panels of railings are disconnected from each other every $8 \mathrm{~m}$. The bridge is supported on pad type elastomeric fixed bearings at the central pylon and similar sliding bearings at the abutments. The sliding bearings were provided to accommodate creep, shrinkage and temperature deformations, and to allow the bridge to move longitudinally in the event of a strong seismic excitation. The bridge is supported by two concrete piles at each abutment and ten concrete piles at the central pylon through a pile cap on their top.

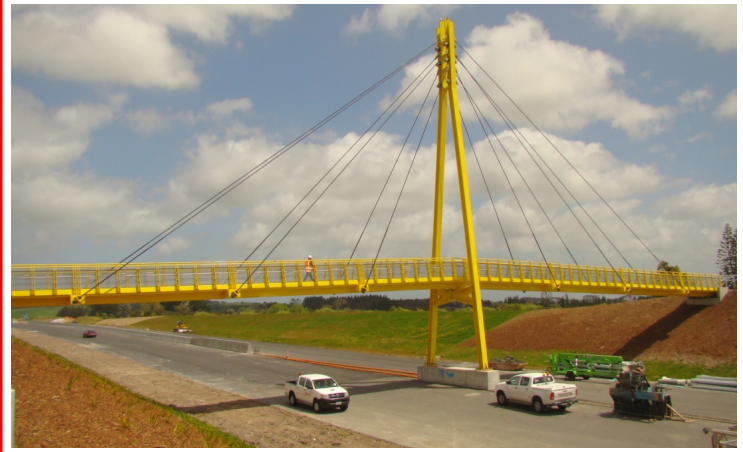

Figure 1. Full scale cable stayed bridge.

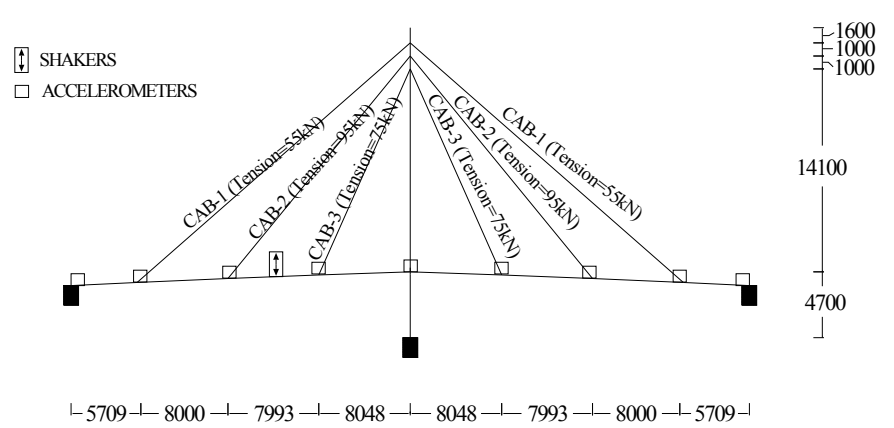

Figure 2. Basic dimensions (in mm) of the bridge, cable forces, and shaker and accelerometer locations.

The main elements to be modeled in case of cable stayed bridges are the deck, pylon, cables and connections of cables and deck. A reasonable representation of bridge deck for box girder sections can be achieved by using beam elements with rigid links joining the cable elements with deck elements ${ }^{23,24}$. In this research, the bridge was modeled in SAP2 $2000^{25}$. The modulus of elasticity of steel was taken as $2.00 \times 10^{11} \mathrm{~N} / \mathrm{m}^{2}$ and for concrete it was taken as $2.80 \times 10^{10} \mathrm{~N} / \mathrm{m}^{2}$. The deck and pylon were modeled using beam type FEs. The beam was discretized into 48 elements, whereas the pylon was discretized into 40 elements. The cables were modeled using catenary elements provided in 
Return to the Manage Active Submissions page at http://spie.org/app/submissions/tasks.aspx and approve or disapprove this submission. Your manuscript will not be published without this approval. Please contact author_help@spie.org with any questions or concerns.

SAP2000 and were discretized into four elements for each cable. Further discretization of these elements did not appreciably affect the natural frequencies and only resulted in an increase in the computational cost. An initial non-linear static analysis was performed to account for the geometric non-linearity caused by the cable sag and that was followed by a linear dynamic analysis to obtain natural frequencies and mode shapes. A linear analysis that uses stiffness from the end of non-linear static analysis for cable stayed structures has been demonstrated to provide accurate results ${ }^{26}$.

Experimental work has been carried out using 15 uni-axial Honeywell QA 750 accelerometers to measure structural response, 3 uni-axial Crossbow MEMS accelerometers to measure shaker input force and a desktop computer fitted with NI DAQ 9203 data acquisition card. Data was collected at a sampling rate of $200 \mathrm{~Hz}$. Three APS Electroseis Model 400 shakers $^{27}$ were used in synchronized mode to impart a combined excitation force of up to $1.2 \mathrm{kN}$ to the structure.

Full scale tests can be conducted by output-only (no measured force) or input-output (measured force) methods. The cable-stayed bridge under study has been tested using both of these methods. The output only test was conducted using jumping to establish the initial estimation of the natural frequencies of the bridge. Two people jumped several times on the bridge in unison to excite the structure and thereafter the bridge was allowed to freely vibrate for two minutes. This was done to establish the range of excitation frequencies for subsequent forced vibration tests. The forced vibration test was then conducted using three dynamically synchronized shakers and their input force was measured using accelerometers mounted on the armature. A sweep sine excitation frequency ranging from 1 to $15 \mathrm{~Hz}$ with the total sweep duration of 466.7 seconds was adopted to adequately excite the structure. The shakers were located away from the deck center line to excite both the vertical and torsional modes. To obtain the natural frequencies in the horizontal direction, the shakers were tilted at 90 degrees. The location of the accelerometers and shakers is shown in Figure 2.

The core of many system identification algorithms is a least square solution which gives a relationship between the input and output of an unknown system. The Numerical Algorithm for Subspace State-Space System Identification (N4SID) ${ }^{28}$, a powerful technique for modal identification in the time domain, is used in this study. The determination of the adequate order of the state space model requires investigation. Theoretically, the system order should be twice the number of the active degrees of freedom. However, due to measurement noise, a higher model order is normally selected to extract the modes of interest with increased confidence. The model order selected for this study ranges from 40 to 80 for vertical and horizontal shaker configurations. As a result, the algorithm identifies some spurious modes which do not represent the actual structural modes. To overcome this problem, stability diagrams are employed. As the system order increases, the structural modes identified by the algorithm should remain stable ${ }^{29}$. Stability tolerances were selected based on the experience and data quality. A tolerance of $1 \%$ for frequency for two subsequent model orders and a value above 0.9 for MAC were selected as the mode stability criteria in this study.

Table 1 summarizes the natural frequencies identified by the N4SID method, and also shows the errors between the experimental frequencies and those identified by the initial FE model. It has been found that the frequencies obtained from the initial FE model differ from the experimental frequencies by as much as $8.6 \%$. However, MAC values are very close to 1, showing that there is a good correlation between the observed and initial FE model mode shapes.

Table 1. Natural frequencies by N4SID, initial FE model, and errors between initial FE model and experimental results.

\begin{tabular}{|ccccc|}
\hline & \multicolumn{3}{c}{ Frequency } & \\
\cline { 2 - 4 } Mode No. & Experiment by N4SID & Initial FE model & Error & \\
\hline 1 & $(\mathbf{H z})$ & $\mathbf{( H z )}$ & $\mathbf{( \% )}$ & MAC \\
2 & 1.64 & 1.66 & 1.22 & 0.999 \\
3 & 1.90 & 1.88 & -1.05 & 0.995 \\
4 & 3.69 & 3.88 & 5.15 & 0.999 \\
5 & 4.86 & 5.28 & 8.64 & 0.999 \\
6 & 5.33 & 5.45 & 2.25 & 0.993 \\
7 & 6.31 & 6.79 & 7.61 & 0.990 \\
8 & 7.42 & 7.76 & 4.58 & 0.980 \\
& 8.32 & 8.66 & 4.09 & 0.993 \\
\hline
\end{tabular}


Return to the Manage Active Submissions page at http://spie.org/app/submissions/tasks.aspx and approve or disapprove this submission. Your manuscript will not be published without this approval. Please contact author_help@spie.org with any questions or concerns.

\section{MODEL UPDATING}

In model updating, dynamic measurements such as natural frequencies and mode shapes are normally correlated with their FE model counterparts to calibrate the FE model. There is a degree of uncertainty in the assessment of the actual properties of the materials used in the full scale structure as well as the most realistic representation of the element stiffness in the initial FE model. The challenge of finding a set of suitable parameters having physical justification necessitates the use of physically significant updating parameters and suitable optimization tools.

The selection of parameters for model updating is a crucial step. Only those parameters should be selected to which the responses are sensitive and whose values are uncertain in the initial model. Inertia parameters were not updated as they are typically less uncertain than stiffness. A sensitivity analysis was then conducted to find out the most influential stiffness parameters. Candidate parameters included cable tensions, cable axial stiffness, bending stiffnesses of the deck and central pylon and bearing stiffness. The parameters eventually selected based on the sensitivity analysis (see Figure 3 ) and engineering insight were the deck stiffness for vertical $\left(\mathrm{K}_{\mathrm{y}, \text { Deck }}\right)$ and horizontal $\left(\mathrm{K}_{\mathrm{x} \text {, Deck }}\right)$ bending, deck torsional stiffness $\left(\mathrm{K}_{\mathrm{t} \text {, Deck }}\right)$, axial stiffness of all cables $\left(\mathrm{K}_{\text {Cable }}\right)$, cable tension for Cab-1 $\left(\mathrm{T}_{\mathrm{Cab}-1}\right)$ and stiffness of bearings $\left(\mathrm{K}_{\text {Bearing }}\right)$. It can be noted from Figure 3 that the sensitivity of $\mathrm{K}_{\mathrm{t} \text {, Deck }}$ and $\mathrm{K}_{\text {Bearing }}$ effect mode 8 in a similar manner signaling the possibility of an ill-posted problem. Therefore a regularization term was introduced into objective function as follows:

$$
\Pi=\Pi_{1}+\Pi_{2}+0.0002 \times\left|\frac{K_{t, \text { Deck }}^{(i)}}{K_{\text {Bearing }}^{(i)}}-\frac{K_{t, \text { Deck }}^{(0)}}{K_{\text {Bearing }}^{(0)}}\right|
$$

where superscript $(i)$ indicates the current iteration step and subscript (0) the initial FE model. The term 0.0002, selected by trial and error, represents the tradeoff between the regularization term and original objective function; a higher value tends to fix the two parameters $K_{t, \text { Deck }}$ and $K_{\text {Bearing }}$ to change together and a lower value allows them to move away from each other during updating. This value has been selected so that the change of the ratio of the two parameters remains approximately within $\pm 10 \%$.

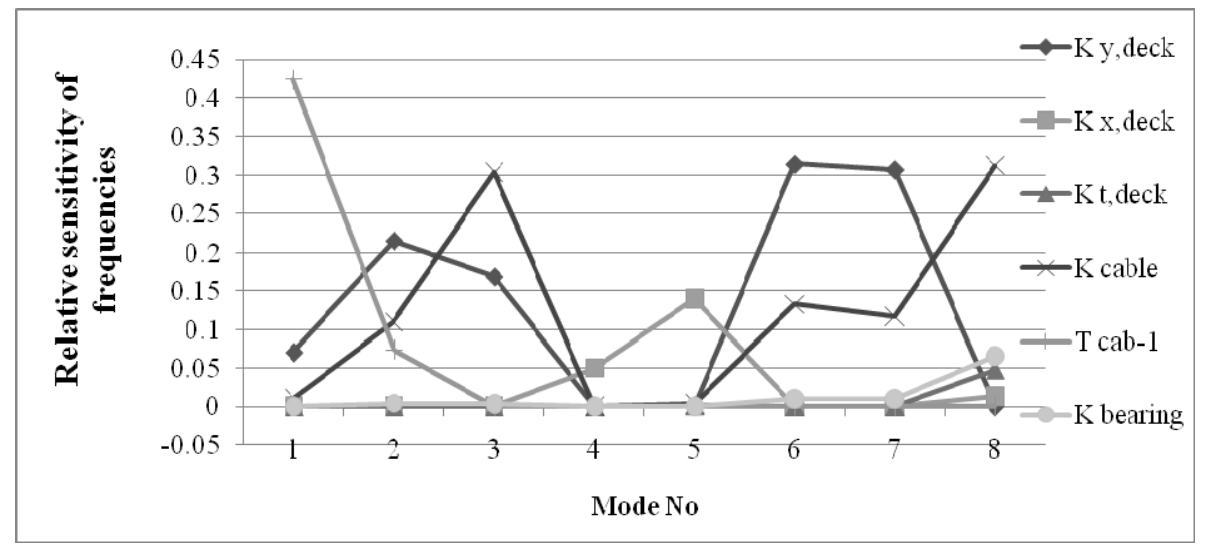

Figure 3. Relative sensitivity of frequencies to selected updating parameters.

It is necessary to estimate the variation bounds of the parameters during model updating and this is normally done using engineering judgment. Different bounds have been used in the past ${ }^{30}$. From the frequency errors in Table 1 , it can be concluded that the initial FE model overestimates the stiffness, therefore the lower bound has been selected as $-40 \%$, and the upper bound has been selected as $+30 \%$ for all the parameters.

A population of 20 particles was used by PSO. On the basis of the extensive study by Clerc and Kennedy ${ }^{31}$, initial values for PSO parameters were set to $\gamma=0.729, c_{1}=1.5$ and $c_{2}=1.5$ (known as the default contemporary PSO variant). The set of parameters for GA was as follows: two-point cross over with a cross over fraction of 0.7 , selection using a tournament technique, and mutation fraction of 0.01 . A value of niche radius was assumed as $0.936^{32}$. The unknown updating parameters were normalized to the range of 0 to 1 . The search was terminated when one of the following three criteria, i.e. minimum threshold value of 0.0025 , maximum number of iterations 200 , or weighted average change in 10 iterations of $2.5 \times 10^{6}$ were satisfied. 
Return to the Manage Active Submissions page at http://spie.org/app/submissions/tasks.aspx and approve or disapprove this submission. Your manuscript will not be published without this approval. Please contact author_help@spie.org with any questions or concerns.

Model updating was attempted by using the six aforementioned unknown parameters related to the stiffness of the bridge. In the first iteration, the solution was obtained by using the basic search algorithm, either PSO or GA, alone without employing SNT. In the subsequent iterations, the SNT algorithm will try to search the space again with niches filled in around the previously found minima. SNT applies a multiplicative factor to the objective function value so that the function value is increased in the vicinity of the known minimum within the niche radius. This essentially forces the algorithm to search for other minima in the search space. However, derating distorts the solution space and may create spurious artificial minima. The algorithm may then be attracted to those spurious minima, a behavior referred to as niche effect. It is therefore imperative to compare the true objective function values and derated values to check for niche effects. Essentially, if the second minimum found is outside the niche radius, the function value with and without SNT would be same and this newly found minimum is accepted. However, if the objective function value with and without SNT is not same, then this implies that the algorithm has been trapped in the modified search space created by derating. Such a solution will not be accepted. The updated solutions in the form of the ratios of updated values to initial values of stiffness parameters are given in Table 2 for PSO with SNT and in Table 3 for GA with SNT. These tables also indicate the objective function values obtained with and without SNT, and the total number of iterations taken by each algorithm to converge.

In Table 2, solution No.1 (shown in bold) has been obtained with PSO only. Further solutions have been obtained by combining PSO with SNT. It can be noted that first solution has the lowest objective function value as compared to the subsequent solutions. Thus, in this case PSO was able to find the global minimum in the first iteration and there are no other minima which give better results in the search space. Furthermore, the two columns comparing the objective function values with and without SNT indicate that solution No.2 suffers from the niche effect. This solution was not accepted and the algorithm continued to search for other four minima in the subsequent runs. Therefore, a total of six solutions are presented in the table to reach the desired number of five correct solutions.

Table 3 presents the results obtained by GA with SNT. A similar strategy as applied previously for PSO with SNT has been used. The first solution (shown in bold) is obtained without application of SNT. The remaining solutions were obtained by combing GA with SNT. It can be seen that solution No.1 obtained with GA (Table 3) alone is in close agreement with the first solution obtained by PSO (Table 2). However, with the application SNT in the subsequent runs, solutions No. 2, 4 and 5 have been found to suffer from niche effects (the objective function values with and without SNT are different) and were therefore discarded. The search process was continued until a total of five acceptable solutions were obtained. The first solution has the smallest value compared to other solutions and therefore shows that GA was also able to successfully identify the global minimum in the first iteration.

Comparing the two approaches, it can be noted that there are more niche effects in GA with SNT than in PSO with SNT. This can be mainly attributed to the approach used by the search algorithms PSO and GA. PSO uses the velocity equation (Equation 1) for searching the solutions where all the particles flying in the search space are essentially influenced by their own best position and the global best position. This enables all the search particles to move in a similar direction. Whereas GA uses a point-wise approach where the chromosomes with the minimum objective function values are favored to survive in the subsequent iterations. The connection between chromosomes is therefore weaker and they tend to cluster around the previous minimum. Also, PSO with SNT has taken less number of iterations as compared to GA with SNT, which suggests that PSO is computationally more efficient.

Only the updating results found by PSO (Table 2) are discussed here in terms of their physical interpretation and significance. The updated frequencies and their errors compared to the experimental results and the updated MAC values are shown in Table 4. It has been found that PSO has given improved results after model updating as compared to the initial frequency estimates. The frequencies and their errors between the updated FE model and experimental results show that maximum frequency difference of $8.62 \%$ in the initial FE model (Table 2) has been reduced to $2.75 \%$ in the updated model. Updated MAC values shown in Table 4 indicate that MACs have either been improved or at least not degraded in the process and all are very close to 1 .

The updated parameters should be physically meaningful; otherwise it is difficult to justify the results with respect to the actual structure. The vertical stiffness of the bridge deck has decreased by $18 \%$, the horizontal stiffness by $18.2 \%$ and the 


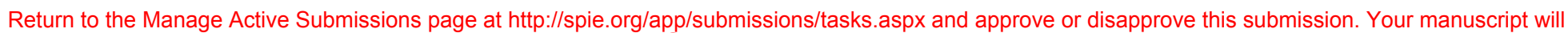
not be published without this approval. Please contact author_help@spie.org with any questions or concerns.

torsional stiffness by $14.5 \%$ respectively. This could be mainly attributed to the fact that the initial model took the cast in-situ concrete slab as fully composite with the steel girder. The updated results reveal that there is only partial composite action between the slab and the steel girder resulting in lower stiffness of the whole deck. The consistent decrease in all the parameters related to the deck stiffness supports this conclusion. It is important to recognize that the original design assumed conservatively a total lack of composite action. The cable axial stiffness has been reduced by $7 \%$ which can be attributed to many factors such as slip in the anchorages or between strands. On the other hand, the increase in cable tension $\mathrm{T}_{\mathrm{Cab}-1}$ by $15 \%$ shows that the cable was likely overstressed during installation.

Table 2. Ratios of updated to initial stiffness values obtained by PSO with SNT and objective function values.

\begin{tabular}{|c|c|c|c|c|c|c|c|c|c|c|}
\hline \multirow{2}{*}{$\begin{array}{c}\text { Solution } \\
\text { No. }\end{array}$} & \multicolumn{6}{|c|}{ Ratio of updated to initial stiffness by PSO with SNT } & \multirow{2}{*}{$\begin{array}{c}\text { Obj. } \\
\text { func. } \\
\text { with SNT }\end{array}$} & \multirow{2}{*}{$\begin{array}{l}\text { Obj. func. } \\
\text { w/out SNT }\end{array}$} & \multirow{2}{*}{ Iterations } & \multirow{2}{*}{ Remarks } \\
\hline & $\mathbf{K}_{\mathbf{y}, \text { Deck }}$ & $\mathbf{K}_{\mathbf{x}, \text { Deck }}$ & $\mathbf{K}_{\mathbf{t}, \text { Deck }}$ & $\mathbf{K}_{\text {Cable }}$ & $\mathbf{T}_{\mathbf{C a b}-1}$ & $\mathbf{K}_{\text {bearing }}$ & & & & \\
\hline 1 & 0.820 & 0.818 & 0.855 & 0.929 & 1.153 & 0.935 & 0.0022 & 0.0022 & 9 & \\
\hline 2 & 0.902 & 1.300 & 1.049 & 0.654 & 1.021 & 0.699 & 0.016 & 0.0036 & 19 & Niche effect \\
\hline 3 & 0.813 & 0.990 & 1.300 & 0.799 & 0.945 & 1.300 & 0.005 & 0.005 & 29 & \\
\hline 4 & 0.803 & 0.870 & 1.300 & 0.997 & 0.831 & 0.600 & 0.014 & 0.014 & 37 & \\
\hline 5 & 0.791 & 0.690 & 0.600 & 1.300 & 0.855 & 0.600 & 0.026 & 0.026 & 36 & \\
\hline 6 & 0.822 & 1.091 & 0.600 & 0.723 & 0.828 & 1.300 & 0.021 & 0.021 & 47 & \\
\hline
\end{tabular}

Table 3. Ratios of updated to initial stiffness values obtained by GA with SNT and objective function values.

\begin{tabular}{|c|c|c|c|c|c|c|c|c|c|c|}
\hline \multirow[b]{2}{*}{ Solution No. } & \multicolumn{6}{|c|}{ Ratio of updated to initial stiffness by GA with SNT } & \multirow{2}{*}{$\begin{array}{l}\text { Obj. } \\
\text { func. } \\
\text { vith SNT }\end{array}$} & \multirow{2}{*}{$\begin{array}{l}\text { Obj. } \\
\text { func. } \\
\text { w/out } \\
\text { SNT }\end{array}$} & \multirow[b]{2}{*}{ Iterations } & \multirow[b]{2}{*}{ Remarks } \\
\hline & $\mathbf{K}_{\mathbf{y}, \text { Deck }}$ & $\mathbf{K}_{\mathbf{x}, \text { Deck }}$ & $\mathbf{K}_{\mathbf{t}, \text { Deck }}$ & $\mathbf{K}_{\text {Cable }}$ & $\mathbf{T}_{\text {Cab-1 }}$ & $\mathbf{K B}_{\text {earing }}$ & & & & \\
\hline 1 & 0.803 & 0.861 & 0.882 & 0.918 & 1.092 & 0.888 & 0.0023 & 0.0023 & 42 & \\
\hline 2 & 0.815 & 0.828 & 0.604 & 0.989 & 1.134 & 0.603 & 0.073 & 0.003 & 66 & Niche effect \\
\hline 3 & 1.003 & 0.766 & 0.613 & 1.076 & 1.143 & 1.292 & 0.016 & 0.016 & 52 & \\
\hline 4 & 0.817 & 0.903 & 0.970 & 0.879 & 1.041 & 1.213 & 0.153 & 0.004 & 32 & Niche effect \\
\hline 5 & 0.812 & 0.870 & 0.607 & 0.943 & 1.028 & 0.861 & 0.219 & 0.005 & 34 & Niche effect \\
\hline 6 & 1.180 & 1.096 & 1.017 & 1.114 & 1.019 & 0.663 & 0.086 & 0.086 & 40 & \\
\hline 7 & 0.809 & 1.296 & 0.739 & 0.636 & 1.050 & 0.722 & 0.036 & 0.036 & 34 & \\
\hline 8 & 0.847 & 0.744 & 0.992 & 1.217 & 0.748 & 0.616 & 0.039 & 0.039 & 35 & \\
\hline
\end{tabular}

Table 4. Updated frequencies and MACs.

\begin{tabular}{|c|c|c|c|c|}
\hline \multirow[t]{2}{*}{ Mode No. } & \multicolumn{3}{|c|}{ Frequencies } & \multirow[t]{2}{*}{ Updated MAC } \\
\hline & $\begin{array}{c}\text { Experiment } \\
(\mathrm{Hz})\end{array}$ & $\begin{array}{c}\text { Updated FE model } \\
(\mathrm{Hz})\end{array}$ & $\begin{array}{l}\text { Error } \\
(\%)\end{array}$ & \\
\hline 1 & 1.64 & 1.68 & 2.18 & 0.999 \\
\hline 2 & 1.89 & 1.88 & -1.29 & 0.995 \\
\hline 3 & 3.69 & 3.68 & -0.44 & 0.998 \\
\hline 4 & 4.86 & 4.92 & 1.09 & 0.989 \\
\hline 5 & 5.33 & 5.27 & -1.29 & 0.987 \\
\hline 6 & 6.31 & 6.33 & 0.21 & 0.999 \\
\hline 7 & 7.42 & 7.22 & -2.75 & 0.991 \\
\hline 8 & 8.32 & 8.36 & 0.58 & 0.992 \\
\hline
\end{tabular}


Return to the Manage Active Submissions page at http://spie.org/app/submissions/tasks.aspx and approve or disapprove this submission. Your manuscript will not be published without this approval. Please contact author_help@spie.org with any questions or concerns.

\section{CONCLUSIONS}

A systematic study of updating of a cable-stayed pedestrian bridge model employing two search algorithms, namely PSO and GA, in combination with SNT is presented in this paper. Following are the conclusions drawn from this study:

1. Five vertical modes, two horizontal modes and one torsional mode of vibration have been successfully identified for the cable-stayed bridge.

2. PSO- and GA-based model updating procedures have been successful in reducing the frequency differences between the initial FE model and the experimental results. The final updated FE model of the bridge is able to produce good agreement with the experimental results while preserving the physical meaning of updated parameters.

3. The results indicate that the slab is not fully composite with the steel girder and the tensions in some cables are more than assumed in design.

4. PSO is found to be computationally more efficient than GA and took less iterations to converge.

5. Niche effects are more pronounced for GA with SNT than PSO with SNT.

6. Combining SNT with PSO and GA gave more confidence that a global minimum is reached in a multidimensional search space.

\section{REFERENCES}

[1] Aktan, A. E., Ellingwood, B. R. and Kehoe, B., "Performance-based engineering of constructed systems," Journal of Structural Engineering, ASCE 133 (3), 311-323 (2007).

[2] Moon, F. L. and Aktan, A. E., "Impacts of epistemic (bias) uncertainty on structural identification of constructed (civil) systems," Shock and Vibration Digest 38 (5), 399-420 (2006).

[3] Beck, J. L. and Jennings, P. C., "Structural identification using linear models and earthquake records," Earthquake Engineering and Structural Dynamics 8 (2), 145-160 (1980).

[4] Catbas, F. N. and Aktan, A. E., "Condition and damage assessment: Issues and some promising indices," Journal of Structural Engineering, ASCE 128 (8), 1026-1036 (2002).

[5] Grimmelsman, K. A., Pan, Q. and Aktan, A. E., "Analysis of data quality for ambient vibration testing of the Henry Hudson bridge," Journal of Intelligent Material Systems and Structures 18 (8), 765-775 (2007).

[6] Salawu, O. S. and Williams, C., "Review of full-scale dynamic testing of bridge structures," Engineering Structures 17 (2), 113-121 (1995).

[7] Schlune, H., Plos, M. and Gylltoft, K., "Improved bridge evaluation through finite element model updating using static and dynamic measurements," Engineering Structures 31 (7), 1477-1485 (2009).

[8] Brownjohn, J. M. W., Xia, P. Q., Hao, H. and Xia, Y., "Civil structure condition assessment by FE model updating: Methodology and case studies," Finite Elements in Analysis and Design 37 (10), 761-775 (2001).

[9] Friswell, M. I. and Mottershead, J. E., Finite element model updating in structural dynamics, Kluwer Academic Publishers, Netherlands, (1995).

[10] Hu, N., Wang, X., Fukunaga, H., Yao, Z. H., Zhang, H. X. and Wu, Z. S., "Damage assessment of structures using modal test data," International Journal of Solids and Structures 38 (18), 3111-3126 (2001).

[11] Hester, D. and González, A., "A wavelet-based damage detection algorithm based on bridge acceleration response to a vehicle," Mechanical Systems and Signal Processing 28 (0), 145-166 (2012).

[12] Deb, K., Optimization for engineering design: Algorithms and examples, Prentice-Hall of India, New Delhi., (1998).

[13] Perera, R. and Torres, R., "Structural damage detection via modal data with genetic algorithms," Journal of Structural Engineering, ASCE 132 (9), 1491-1501 (2006).

[14] Raich, A. M. and Liszkai, T. R., "Improving the performance of structural damage detection methods using advanced genetic algorithms," Journal of Structural Engineering, ASCE 133 (3), 449-461 (2007).

[15] Levin, R. I. and Lieven, N. A. J., "Dynamic finite element model updating using simulated annealing and genetic algorithms," Mechanical Systems and Signal Processing 12 (1), 91-120 (1998). 
Return to the Manage Active Submissions page at http://spie.org/app/submissions/tasks.aspx and approve or disapprove this submission. Your manuscript will not be published without this approval. Please contact author_help@spie.org with any questions or concerns.

[16] Saada, M. M., Arafa, M. H. and Nassef, A. O., "Finite element model updating approach to damage identification in beams using particle swarm optimization," Proc. 34th Design Automation Conference, ASME, 522-531 (2008).

[17] Begambre, O. and Laier, J. E., "A hybrid particle swarm optimization - simplex algorithm (PSOS) for structural damage identification," Advances in Engineering Software 40 (9), 883-891 (2009).

[18] Kennedy, J. and Eberhart, R., "Particle swarm optimization," Proc. IEEE International Conference on Neural Networks, 1942-1948 (1995).

[19] Holland, J., Adaptation in natural and artificial systems, MIT Press, Cambridge, Mass, (1975).

[20] Beasley, D., Bull, D. R. and Martin, R. R., "A sequential niche technique for multimodal function optimization," Evolutionary Computation 1 (2), 101-125 (1993).

[21] Möller, P. W. and Friberg, O., "Updating large finite element models in structural dynamics," AIAA Journal 36 (10), 1861-1868 (1998).

[22] AS/NZS, AS/NZS3679 Part-1 Structural steel hot rolled bars and sections, Standards Australia, Sydney and Standards New Zealand, Wellington, (1996).

[23] Ren, W. X. and Peng, X. L., "Baseline finite element modeling of a large span cable-stayed bridge through field ambient vibration tests," Computers and Structures 83 (8-9), 536-550 (2005).

[24] Chang, C. C., Chang, T. Y. P. and Zhang, Q. W., "Ambient vibration of long-span cable-stayed bridge," Journal of Bridge Engineering, ASCE 6 (1), 46-53 (2001).

[25] Computers and Structures, SAP2000 Structural analysis program, Ca, 2009.

[26] Abdel-Ghaffar, A. M. and Khalifa, M. A., "Importance of cable vibration in dynamics of cable-stayed bridges," Journal of Engineering Mechanics, ASCE 117 (11), 2571-2589 (1991).

[27] APSDynamics, Dynamic Electroseis shaker model 400. Http://www.Apsdynamics.Com/ 2012.

[28] Overschee, P. V. and Moor, B., Subspace identification for the linear systems: Theory-implementation, Kluwer Academic Publishers, Netherlands, (1996).

[29] Bodeux, J. B. and Golinval, J. C., "Application of ARMAV models to the identification and damage detection of mechanical and civil engineering structures," Smart Materials and Structures 10 479-489 (2001).

[30] Jaishi, B. and Ren, W. X., "Structural finite element model updating using ambient vibration test results," Journal of Structural Engineering, ASCE 131 (4), 617-628 (2005).

[31] Clerc, M. and Kennedy, J., "The particle swarm - explosion, stability, and convergence in a multidimensional complex space," IEEE Transactions on Evolutionary Computation 6 (1), 58-73 (2002).

[32] Deb, K., Genetic algorithms in multimodal function optimization, M.S. thesis (rep. 89002), The University of Alabama, Tuscaloosa, AL, (1989). 